\title{
Voxel-Wise Displacement as Independent Features in Classification of Multiple Sclerosis
}

\author{
Min Chen ${ }^{1,2}$, Aaron Carass ${ }^{1}$, Daniel S. Reich ${ }^{2}$, Peter A. Calabresi ${ }^{3}$, \\ Dzung Pham ${ }^{4}$, and Jerry L. Prince ${ }^{1}$ \\ ${ }^{1}$ Image Analysis and Communications Laboratory, Dept. of ECE, Johns Hopkins University \\ ${ }^{2}$ Translational Neuroradiology Unit, National Institute of Neurological Disorders and Stroke \\ ${ }^{3}$ Department of Neurology, Johns Hopkins School of Medicine \\ ${ }^{4}$ Image Processing Core, Center for Neuroscience and Regenerative Medicine
}

\begin{abstract}
We present a method that utilizes registration displacement fields to perform accurate classification of magnetic resonance images (MRI) of the brain acquired from healthy individuals and patients diagnosed with multiple sclerosis (MS). Contrary to standard approaches, each voxel in the displacement field is treated as an independent feature that is classified individually. Results show that when used with a simple linear discriminant and majority voting, the approach is superior to using the displacement field with a single classifier, even when compared against more sophisticated classification methods such as adaptive boosting, random forests, and support vector machines. Leave-one-out cross-validation was used to evaluate this method for classifying images by disease, MS subtype (Acc: $77 \%-88 \%$ ), and age (Acc: $96 \%-100 \%)$.
\end{abstract}

Keywords: Image registration, Magnetic resonance imaging, Classification, Multiple Sclerosis.

\section{INTRODUCTION}

Discriminant analysis using classifiers derived from magnetic resonance images (MRI) is an important tool in the study of neurological diseases. Several MR features have been used to classify between healthy controls and patients with disease ${ }^{1-5}$. Our work here focuses primarily on classification using features derived from displacement fields learned by deformably registering a MR image to a template. One advantage to using these deformation based features is the ability to infer group shape differences from the classifier. For example, Golland et $a l .{ }^{6}$ demonstrated this by analyzing the gradient of the classifier function, which described a "discriminative direction" for hippocampus shape change between populations. Similarly, Qiu et al. ${ }^{7}$ used a linear discriminant on surface deformations and analyzed the normal of the separating hyperplane to characterize population differences.

Related to our approach is a group of methods known as deformation-based morphometry (DBM), which finds regions with significant population differences by observing volume changes through the Jacobian of the displacement field ${ }^{8}$ or performing a significance test on the population means of the displacements ${ }^{9,10}$. Our method differs from DBM in that we are focused on building a classifier between two populations, while DBM is primarily interested in localizing areas with significant differences. One advantage of our approach is that the classifiers found by our method can potentially be used to model the direction of change between two populations, such as in Golland et al. ${ }^{6}$

\section{NEW WORK PRESENTED}

While a number of works have explored using deformation based features for classification, these methods have all traditionally used only a single classifier on these features. For example, Lao et al. ${ }^{11}$ performed classification using support vector machines (SVM) with volume-preserving features calculated from the Jacobian of the displacement fields. Golland et al. ${ }^{6}$ also used SVM, but used distance transforms to construct the feature vectors. Rueckerk et al. ${ }^{12}$ performed principle component analysis on the control points of b-spline representations of the

Further author information, send correspondence to Min Chen (mchen55@jhu.edu)

Medical Imaging 2013: Image Processing, edited by Sebastien Ourselin, David R. Haynor, Proc. of SPIE Vol. 8669, 86690K · @ 2013 SPIE · CCC code: 1605-7422/13/\$18

doi: $10.1117 / 12.2007150$

Proc. of SPIE Vol. $866986690 \mathrm{~K}-1$ 
displacement fields. We present an approach that moves away from these single classifier frameworks. Instead, we use displacement field vectors as independent features that are classified individually on each voxel. This creates a set of weak classifiers that we then combine together to produce our final classification. Our approach follows in the spirit of multiple classifier machine learning techniques such as boosting ${ }^{13}$ and classifier fusion. ${ }^{14}$

\section{METHODS}

\subsection{Voxel-wise Displacement Vectors as Independent Features}

Given a subject image, $S(x)$, and a template image, $T(x)$, we use deformable registration to find a mapping $v: \mathbb{R}^{3} \rightarrow \mathbb{R}^{3}$, such that $S(x+v(x)) \simeq T(x)$. This mapping is generally found by maximizing a cost function that describes the similarity between $S(x+v(x))$ and $T(x)$. Popular examples of such similarity functions include mutual information, cross correlation, and sum of squared differences. ${ }^{15}$

In practice, the mapping $v$ is represented as a displacement field that consists of a 'pull-back' vector located at each voxel in the template image. These vectors describe the direction and magnitude from a voxel in the template image to its corresponding location in the subject image. For a group of subjects, these displacement fields can be used to approximate the morphological difference between each subject and the template. Since the displacement vectors are all rooted in the template space, the subjects' displacements at the same voxel can be analyzed as a group. Figure 1 shows the registration result and the three components of the displacement field for two subjects registered to the same template.

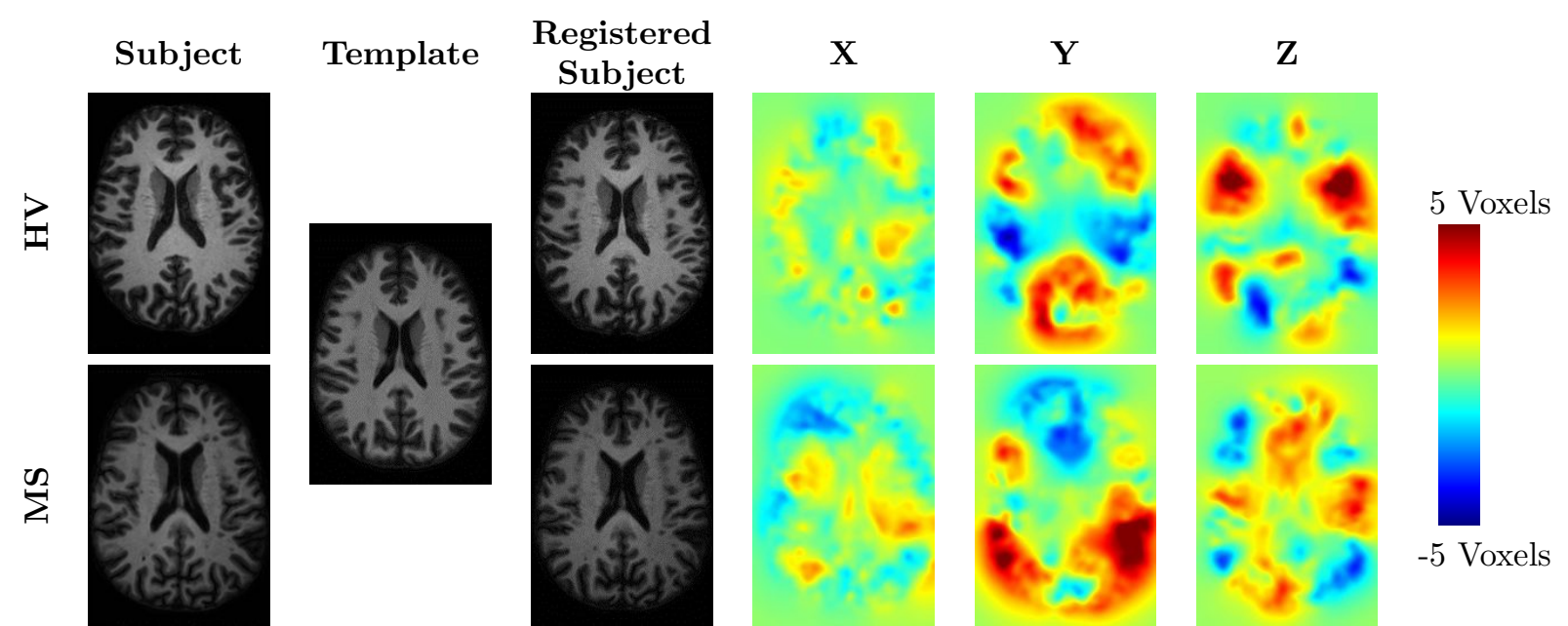

Figure 1. Shown are subject, template, registered subject, and the X, Y, and Z components of the displacement field images for a HV and MS subject.

Due to the regularization in the registration and the structural relationship between different subjects, we know that adjacent voxels in a displacement field are spatially dependent. However, in this method, we propose treating the vectors at each voxel as independent features. We do this by building a separate classifier at each voxel and then aggregating the collection using a simple fusion technique.

\subsection{Classification Using Linear Discriminants and Majority Voting}

We are given a set of $N$ subject images, each with a classification, $y \in\{-1,1\}$, and a displacement field, $v$, relative to the same template image. We designate the pairs, $\left\{v_{1}, y_{1}\right\},\left\{v_{2}, y_{2}\right\}, \ldots,\left\{v_{N}, y_{N}\right\}$, as the training set for our classification algorithm. We are interested in using this training set to build a classifier, $H(v)$, that is able to use a displacement field to correctly estimate the classification of an unseen subject image. We propose first building a weak classifier at each voxel $x$ :

$$
h_{x}(\cdot)=\underset{h(\cdot)}{\arg \min } \sum_{i=1}^{N} I\left(y_{i} \neq h\left(v_{i}(x)\right)\right),
$$


where $I$ is the indicator function, and $h$ can be any standard classifier such that $h: \mathbb{R}^{3} \rightarrow\{-1,1\}$. For the purposes of demonstration (with the added benefits of speed and simplicity) we use Fisher's linear discriminate ${ }^{16}$ as our voxel-wise classifier. Clearly, we do not expect the classifier at any particular voxel to be very accurate for classifying the whole image. However, several works ${ }^{17}$ have shown that a large number of weak classifiers can be combined together to perform a much more accurate classification. To perform this classifier fusion, we first compute the signed training error, $\alpha(x)$, at each voxel,

$$
\alpha(x)=\frac{1}{N} \sum_{i=1}^{N} I\left(y_{i}=h_{x}\left(v_{i}(x)\right)\right)-0.5 .
$$

These errors are then used to weight the classification from each of the weak classifiers, which are summed together to form $H(v)$. Given a displacement field, $\tilde{v}$, from an unseen subject, we estimate its classification with,

$$
H(\tilde{v})=\operatorname{sign}\left(\sum_{x} \alpha(x) h_{x}(\tilde{v}(x))\right) .
$$

This formulation is simply a weighted majority voting of the weak classifiers, $h_{x}$, over all the voxels in the image. Again, we choose this approach purely for brevity. More sophisticated forms of classifier fusion ${ }^{18}$ could potentially be used to improve on this method.

\subsection{Preprocessing and Displacement Field Creation}

All the brain images were extracted from MR head scans using SPECTRE ${ }^{19}$ to remove all non-brain voxels. For the purpose of this paper we are only interested in using local morphological differences for the classification. Hence, rotational, translational and size differences were removed by affinely registering ${ }^{20}$ each image to a common space.

The displacement fields used in our classification were constructed by deformably registering each subject image to a common template. To minimize potential bias, the template was chosen to be an average brain created from all the subjects through the average template building tool provided in $\mathrm{ANTS}^{20}$. The deformable registrations were performed using $\mathrm{SyN}^{21}$, a symmetric diffeomorphic fluid based registration, which was highly ranked in both accuracy and robustness in a comparison among publicly available registration algorithms ${ }^{22}$.

\section{RESULTS AND DISCUSSION}

To evaluate our approach, the template was built using 78 brain images-36 age-matched healthy volunteers (HV) as controls and 42 MS subjects (with subgroups: 29 Relapsing Remitting (RRMS); 8 Secondary Progressive (SPMS), 4 Clinically Isolated Syndrome (CIS), and 1 Primary Progressive (PPMS)). The images are MPRAGE $\left(\mathrm{TR}=10 \mathrm{~ms} ; \mathrm{TE}=6 \mathrm{~ms} ; \alpha=8^{\circ}\right.$; resolution $\left.=0.828 \times 0.828 \times 1.1 \mathrm{~mm}\right)$ acquired on a Philips $3 \mathrm{~T}$ scanner.

The displacement fields and subject classifications were used as inputs for five leave-one-out, cross-validation experiments. In each case the classification performance was compared between the proposed method and three standard machine learning tools from the Weka ${ }^{23}$ software package - Random Forest ${ }^{17}$ ("RandomForest" in Weka), Support Vector Machines ${ }^{24}$ ("SMO" in Weka), and Adaptive Boosting ${ }^{13}$ ("AdaBoostM1" in Weka). For the three standard tools, the displacement fields were reshaped into one-dimensional vectors so that they could be used directly in the classifiers. The following sections will describe the details for these experiments.

\subsection{Downsampling and Parameter Tuning}

While not a problem for our proposed method, the large size of the displacement fields was a major concern when used with the three standard classification tools. Each displacement field had a three dimensional vector at each of roughly eight million voxels $(256 \times 256 \times 120)$. For the larger experiments, this meant the three standard tools took several hours to a full day just to train on the data. In comparison, our approach took on average 2-3 minutes to train and never exceeded more than 10 minutes. We believe this computational efficiency comes from the linear complexity of our method (with respect to the number of voxels), and overall less memory access 
and usage, since the majority of the computation is separable at each voxel. Although not implemented for this paper, this separability also makes our approach easily parallelizable if faster computation is necessary.

In order to run the comparison in a reasonable time, each displacement field was downsampled in each dimension before being classified by the four algorithms. This is a technique that is often performed to make the size of such displacement fields more manageable ${ }^{25,26}$. In each experiment, the displacement field was downsampled by both a factor of two in each direction and also eight in each direction.

Several parameters for the three standard tools were manually selected to give the best average performance over all the experiments. These parameters were: for random forest - "Number of Trees", for support vector machine - "Complexity Constant", and for adaptive boosting - "Number of Iterations". All other parameters were left as the default settings from Weka. We recognize that the accuracy for these methods could potentially be improved by tuning additional parameters and using more complex parameter selection approaches. However, we also note that no tuning was performed at all for our proposed approach.

\subsection{Classification of Age}

Two age classification experiments were performed using just the healthy volunteer data: the oldest five HV (avg. age: 55.5) vs. the youngest five HV (avg. age: 22.4), and the oldest $11 \mathrm{HV}$ (avg. age: 49.9) vs. the youngest 11 HV (avg. age: 23.6). We see from Table 1 that our classifier is highly accurate for both classifications. Compared to the three standard approaches, our method was on average 10-20\% more accurate. As we would expect, the accuracy dropped as the mean age between the populations became closer.

\subsection{Classification of Multiple Sclerosis}

Three disease based classifications were performed using the data: the 29 RRMS patients vs. 29 age-matched $\mathrm{HV}$, the eight SPMS patients vs. eight age-matched HV, and all $42 \mathrm{MS}$ patients vs. all $36 \mathrm{HV}$. Table 1 shows that the proposed approach, again, had the highest accuracy in each of these classification experiments. We also observe that, for each of the experiments, the level of accuracy matched our expectation for the MS subtype being compared.

Relapsing-remitting MS (RRMS) is considered an early stage subtype of MS that is characterized by sporadic attacks. Hence, we would expect the brain morphology for the mild cases to be very similar to the healthy volunteers, and therefore more difficult to classify. In contrast, secondary-progressive MS (SPMS) is a late stage subtype that follows RRMS, and is characterized by a consistent progression of disability. For several of these patients, we could visually see significant morphological change in their brain MRI. This contributes to why the overall accuracy is fairly high for that experiment. Lastly, for the "all MS vs. all HV" experiment, we are actually grouping together four different subtypes of MS that could have entirely separate morphological characterizations. This makes such a classification task extremely difficult, and hence the low accuracy result.

Table 1. Experiment results on deformation fields downsampled either by a factor of two or eight in each dimension. Shown are the classification accuracy of AdaBoost (AB), Random Forest (RF), and support vector machine (SVM) compared to the proposed voxel-wise displacement classifier (VDC). For each case, the downsampling factor is indicated as a suffix to the method name.

\section{Experiment}

5 Youngest vs. 5 Oldest 11 Youngest vs. 11 Oldest All MS vs. All Controls SPMS vs. Controls RRMS vs. Controls
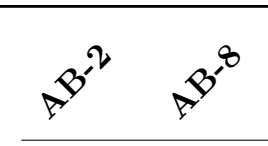

$0.40 \quad 0.50$

$0.5 \quad 0.64$

$0.60 \quad 0.56$

$\begin{array}{ll}0.56 & 0.88\end{array}$

$0.48 \quad 0.54$

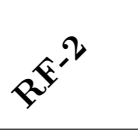

0.90

0.64

0.62

0.63

0.52

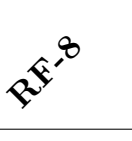

0.70

0.41

0.64

0.63

0.48

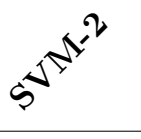

0.70

0.50

0.50

0.44

0.41

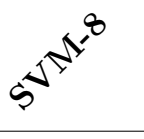

0.70

0.50

0.51

0.38

0.41

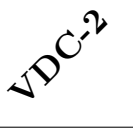

1.00

0.96

0.73

0.88

0.77

$9^{8.8}$
1.00
0.96
0.76
0.88
0.79




\subsection{Comparison Against Existing Literature}

Deformation-based age classification have been reported in several work ${ }^{11,27}$. Although different data was used, we see that the accuracy for our method is comparable, if not superior, to the 70-98\% accuracy reported by these methods. We note, however, that these approaches are not direct comparison to our method. Their primarily focus was on using sophisticated feature selection to restrict the feature space for the classifier. In contrast, our method is focused on improving the classifier itself. The two are complements of each other, and it would be rather straightforward to substitute the standard classifier (i.e. SVM) in these approaches with our method in order to gain the benefits from both methods.

Several work have reported on discriminant analysis of MS and its subtypes, but (to our knowledge) only when using unconventional MR acquisitions such as magnetization transfer ratio (MTR) images ${ }^{1}$. Since the data used were significantly different, we can only make marginal comparisons with our results. For example, we see that our accuracy is overall lower than the MTR histogram approach presented by Dehmeshki et al. ${ }^{1}$, which achieved a classification accuracy of $86-92 \%$ for various MS subtypes. Nevertheless, our method performed surprisingly close given that we used a standard MR acquisition that lacked the features provided by these specialize scans.

\section{CONCLUSION}

We have presented a deformation based classifier that breaks away from the standard single classifier approach by assembling individual weak classifiers at each voxel to generate a final classification. Our approach produced high accuracy when classifying morphological differences in age and various subtypes of MS. We showed that our classifier is superior to using standard classifiers directly on the displacement fields, and that our classification accuracy is comparable to those reported when using sophisticated feature selection methods and unconventional MR modalities. In future work, we intend to utilize the classifiers learned from our method to perform shape based statistical modeling, and potentially infer the direction of change between the various MS populations.

\section{ACKNOWLEDGEMENTS}

This work was supported in part by NIH/NINDS grant R01-NS070906, the Intramural Research Program of NINDS, and the National MS Society (NMSS).

\section{REFERENCES}

[1] Dehmeshki, J., Barker, G. J., and Tofts, P. S., "Classification of disease subgroup and correlation with disease severity using magnetic resonance imaging whole-brain histograms: application to magnetization transfer ratios and multiple sclerosis," IEEE Trans. Med. Imag. 21(4), 320-31 (2002).

[2] Lin, F., Yu, C., et al., "Discriminative analysis of relapsing neuromyelitis optica and relapsing-remitting Multiple Sclerosis based on Two-Dimensional histogram from diffusion tensor imaging," NeuroImage 31(2), 543-549 (2006).

[3] DeCarli, C., Murphy, D. G. M., et al., "Discriminant analysis of MRI measures as a method to determine the presence of dementia of the Alzheimer type," Psychiatry Research 57(2), 119-130 (1995).

[4] Kontos, D., Megalooikonomou, V., et al., "Extraction of discriminative functional mri activation patterns and an application to alzheimer's disease," in [in Proc. of the 7th International Conference on Medical Image Computing and Computer Assisted Intervention (MICCAI 2004), Lecture Notes in Computer Science], 727735, Springer-Verlag (2004).

[5] Fan, Y., Shen, D., Gur, R. C., Gur, R. E., and Davatzikos, C., "COMPARE: Classification of Morphological Patterns Using Adaptive Regional Elements," IEEE Trans. Med. Imag. 26(1), 93-105 (2007).

[6] Golland, P., Grimson, W. E. L., Shenton, M. E., and Kikinis, R., "Detection and analysis of statistical differences in anatomical shape," Medical Image Analysis 9(1), 69-86 (2005).

[7] Qiu, A., Younes, L., Miller, M. I., and Csernansky, J. G., "Parallel transport in diffeomorphisms distinguishes the time-dependent pattern of hippocampal surface deformation due to healthy aging and the dementia of the Alzheimer's type," NeuroImage 40(1), 68-76 (2008). 
[8] Davatzikos, C., "Brain morphometrics using geometry-based shape transformations," in [In Proceedings of Workshop on Biomedical Image Registration], (1999).

[9] Gaser, C., Volz, H. P., Kiebel, S. J., Riehemann, S., and Sauer, H., "Detecting structural changes in whole brain based on nonlinear deformations-Application to schizophrenia research," NeuroImage 10(2), 107-113 (1999).

[10] Cao, J. and Worsley, K. J., "The detection of local shape changes via the geometry of Hotellings $T^{2}$ fields," The Annals of Statistics 27(3), 925-942 (1999).

[11] Lao, Z., Shen, D., Xue, Z., Karacali, B., Resnick, S. M., and Davatzikos, C., "Morphological classification of brains via high-dimensional shape transformations and machine learning methods," NeuroImage 21(1), $46-57$ (2004).

[12] Rueckert, D., Frangi, A. F., and Schnabel, J. A., "Automatic construction of 3-D statistical deformation models of the brain using nonrigid registration," IEEE Trans. Med. Imag. 22(8), 1014-1025 (2003).

[13] Freund, Y. and Schapire, R. E., "A Decision-Theoretic Generalization of on-Line Learning and an Application to Boosting," Journal of Computer and System Sciences 55(1), 119-139 (1997).

[14] Kuncheva, L. I., "A theoretical study on six classifier fusion strategies," IEEE Trans. on Pattern Anal. Machine Intell. 24(2), 281-286 (2002).

[15] Pluim, J. P. W., Maintz, J. B. A., and Viergever, M. A., "Mutual Information Based Registration of Medical Images: A Survey," IEEE Trans. Med. Imag. 22(8), 986-1004 (2003).

[16] Fisher, R. A., "The Use of Multiple Measurements in Taxonomic Problems," Annals of Eugenics 7(2), 179-188 (1936).

[17] Breiman, L., "Random Forests," Machine Learning 45(1), 5-32 (2001).

[18] Kuncheva, L. I., Bezdek, J. C., and Duin, R. P., "Decision templates for multiple classifier fusion: an experimental comparison," Pattern Recognition 34(2), 299 - 314 (2001).

[19] Carass, A., Cuzzocre, J., Wheeler, M. B., Bazin, P. L., Resnick, S. M., and Prince, J. L., "Simple paradigm for extra-cerebral tissue removal: Algorithm and analysis," NeuroImage 56(4), 1982-1992 (2011).

[20] Avants, B. B., Tustison, N. J., Song, G., Cook, P. A., Klein, A., and Gee, J. C., "A reproducible evaluation of ANTs similarity metric performance in brain image registration," NeuroImage 54(3), 2033-2044 (2011).

[21] Avants, B. B., Epstein, C. L., Grossman, M., and Gee, J. C., "Symmetric diffeomorphic image registration with cross-correlation: evaluating automated labeling of elderly and neurodegenerative brain," Medical Image Analysis 12(1), 26-41 (2008).

[22] Klein, A., Ghosh, S. S., and et al., "Evaluation of volume-based and surface-based brain image registration methods," NeuroImage 51(1), 214-220 (2010).

[23] Hall, M., Frank, E., Holmes, G., Pfahringer, B., Reutemann, P., and Witten, I. H., "The weka data mining software: an update," SIGKDD Explor. Newsl. 11, 10-18 (Nov. 2009).

[24] Cortes, C. and Vapnik, V., "Support-vector networks," Machine Learning 20, 273-297 (1995).

[25] Kim, M. J., Kim, M. H., and Shen, D., "Learning-based deformation estimation for fast non-rigid registration," in [CVPR Workshop], (2008).

[26] Long, X. and Wyatt, C., "Structural template formation with discovery of subclasses," in [Society of PhotoOptical Instrumentation Engineers (SPIE) Conference Series], Society of Photo-Optical Instrumentation Engineers (SPIE) Conference Series 7623 (Mar. 2010).

[27] Golland, P., Fischl, B., and et al., "Discriminative analysis for image-based studies," in [in Proc. of the 5th International Conference on Medical Image Computing and Computer Assisted Intervention (MICCAI 2002), Lecture Notes in Computer Science], 2488, 508-515 (2002). 